\title{
Parálisis de cuerda vocal secundaria a intubación endotraqueal y su manejo. Revisión en relación a dos casos clínicos
}

\section{Vocal cord paralysis secondary to endotracheal intubation and its management. Review in relation to two clinical cases}

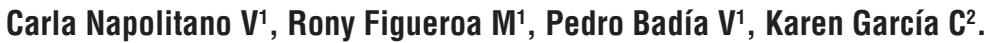

\begin{abstract}
RESUMEN
La parálisis de cuerda vocal secundaria a intubación endotraqueal es una complicación poco frecuente de la anestesia general. Se manifiesta con disfonía precoz luego de la extubación, y en ocasiones se asocia a aspiración. A continuación se presenta el reporte de dos pacientes sometidos a cirugía abdominal con anestesia general e intubación endotraqueal, que presentan una parálisis cordal unilateral en el posoperatorio inmediato.

En ambos pacientes se realiza una laringoplastía de inyección con ácido hialurónico (Restylane $\left.{ }^{\circledR}\right)$, con buenos resultados vocales. Uno de los casos tiene seguimiento a lo largo de dos años. Se describe esta patología y su manejo mediante una revisión bibliográfica.
\end{abstract}

Palabras clave: Parálisis unilateral de cuerda vocal, intubación endotraqueal, disfonía, laringoplastía de inyección.

\begin{abstract}
Vocal fold paralysis posterior to endotracheal intubation is a very uncommon complication of the general anestesia. The symptoms includes hoarseness posterior to the extubation, and some times aspiration occurs. In this paper we present the report of two cases of abdominal surgery with general anesteshia and endotraqueal intubation procedures. Both subjects developed unilateral vocal fold paralysis after the process.

The patients were treated with wedilization injection with Restylane ${ }^{\circledR}$, satisfactory voice 's results were achieved. One of them has an observational time of two years. We describe this infrequent pathology and the treatment through literature review.

Key words: Unilateral vocal fold paralysis, endotracheal intubation, hoarseness, medialization injection.
\end{abstract}

1 Médicos del Departamento de Otorrinolaringología, Pontificia Universidad Católica de Chile.

2 Interna de Medicina, Pontificia Universidad Católica de Chile. 


\section{INTRODUCCIÓN}

La parálisis cordal unilateral secundaria a intubación endotraqueal es una complicación inhabitual de la anestesia general, que se manifiesta clínicamente por disfonía ${ }^{1}$. Existen reportes de casos en la literatura pero éstos son limitados ${ }^{1}$. Diferentes factores se han asociado a su ocurrencia, tales como edad y comorbilidades del paciente, así como posición y presión del cuff' del tubo endotraqueal ${ }^{2,3}$. Es un cuadro de curso habitualmente benigno ${ }^{1}$. Las alternativas terapéuticas incluyen la terapia fonoaudiológica, laringoplastía de inyección con diferentes materiales, y tiroplastía de medialización $n^{4-5}$.

El objetivo de este trabajo es presentar dos casos clínicos de parálisis cordal unilateral secundaria a intubación endotraqueal, su manejo con laringoplastía de inyección precoz con ácido hialurónico (Restylane ${ }^{\circledR}$ ), junto con una revisión de la literatura.

\section{CASOS CLÍNICOS}

\section{Caso 1}

Hombre de 38 años, con antecedente de tabaquismo activo, sometido de urgencia a un drenaje laparoscópico de un absceso pélvico secundario a apendicitis aguda. Dos días después de la cirugía presenta disfonía de inicio súbito, sin tos ni disfagia. Se realiza una nasofaringolaringoscopía (NFC) que muestra cuerda vocal izquierda inmóvil en posición lateral, con signo del "empujón" presente ("jostle sign"), asociada a importante hiato fonatorio. La cuerda vocal derecha presenta movilidad conservada en aducción y abducción. Se complementa estudio con tomografía computarizada (TC) de cuello y tórax, que resultan normales.

Los puntajes de las encuestas de autopercepción de la voz fueron: RSI (Reflux Symptom Index) de 5, VRQOL (Voice Related Quality of Life) de 35, VHI-10 (Voice Handicap Index) de 40, la deglución fue 0 y la autopercepción fue pobre. Dada la insuficiencia glótica sintomática que presenta el paciente, se realiza una laringoplastía de inyección con ácido hialurónico (Restylane ${ }^{\circledR}$ ) dos semanas posterior al inicio del cuadro. El procedimiento se realizó bajo visión nasofibroscópica con paciente vigil y anestesia local, y se utilizó la técnica de inyección percutánea con abordaje transtirohioideo, logrando cierre completo del hiato fonatorio. En el control 3 días posterior a la laringoplastía de inyección, la nasofaringolaringoscopía se informa con cuerda vocal izquierda inmóvil en posición paramediana, movilidad de cuerda vocal derecha conservada en aducción y abducción, hiperfunción supraglótica secundaria, especialmente uso de bandas ventriculares. Mínimo hiato fonatorio posterior. Comisura posterior normal. Luego completa terapia fonoaudiológica. Dos años posterior a la parálisis cordal secundaria a intubación endotraqueal, el paciente se encuentra asintomático. Evaluación nasofibroscópica revela cuerda vocal inmóvil en posición paramediana y movilidad de cuerda vocal derecha conservada tanto en aducción como abducción y compensando cierre glótico a izquierda (Figura 1).
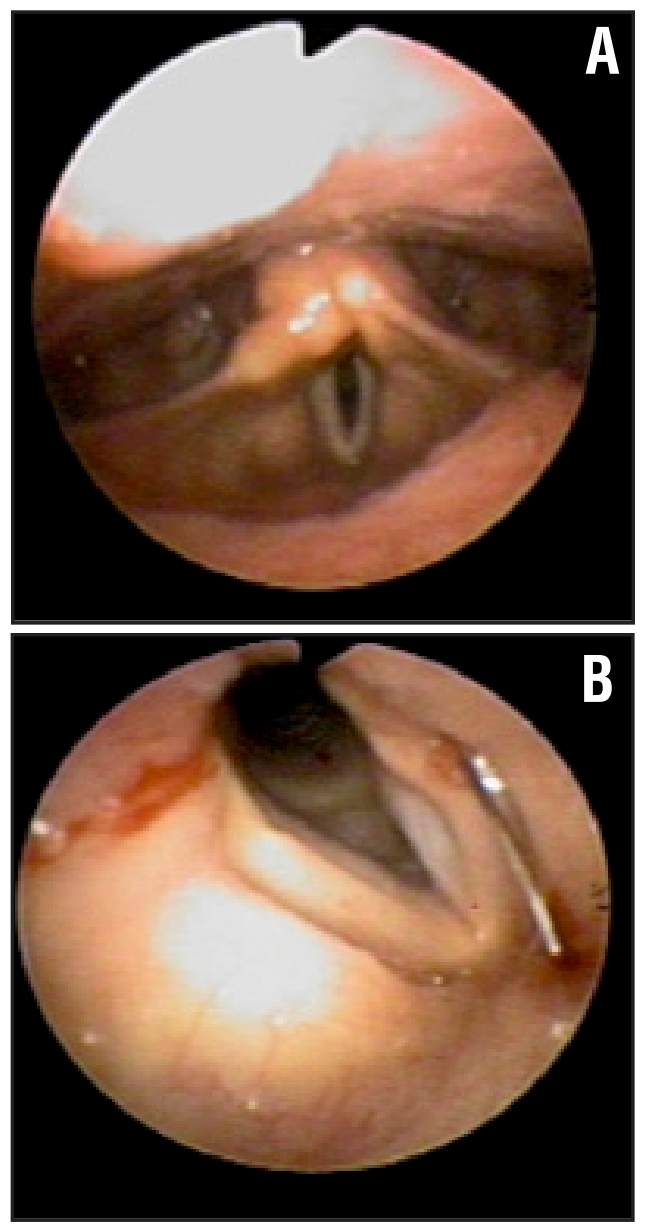

Figura 1. A). Imagen prelaringoplastía de inyección con ácido hialurónico. B). Procedimiento laringoplastía de inyección con ácido hialurónico. 


\section{Caso 2}

Hombre de 53 años, sometido a una sigmoidectomía. Presenta disfonía 24 horas posterior a la cirugía, sin otros síntomas. No se describe intubación difícil durante la anestesia. El paciente es evaluado mediante nasofaringolaringoscopía, que muestra inmovilidad de la cuerda vocal izquierda en posición lateral, con signo del "empujón"
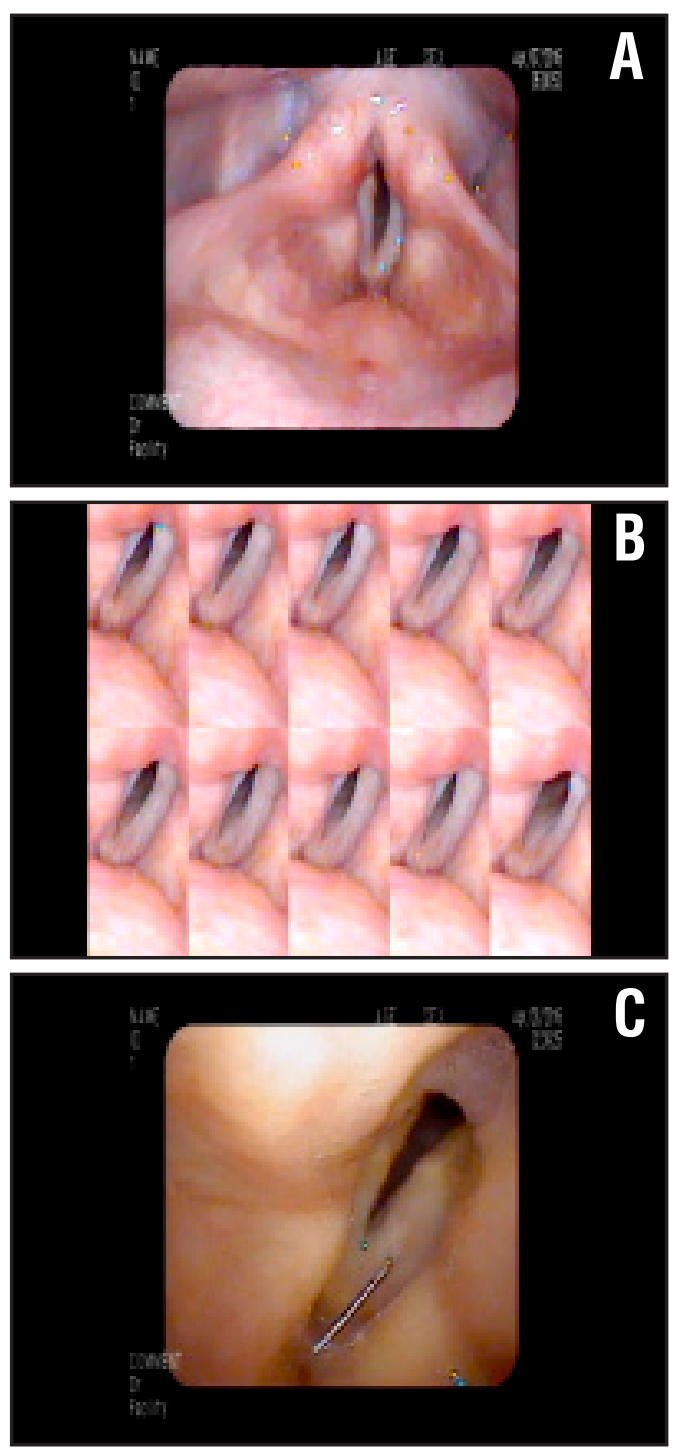

Figura 2. A). Imagen prelaringoplastía de inyección con ácido hialurónico. C). Procedimiento laringoplastía de inyección con ácido hialurónico. presente ("jostle sign"), asociada a importante hiato fonatorio longitudinal y en altura. La cuerda vocal derecha presenta movilidad conservada en aducción y abducción. Se TC de cuello y tórax, que resultan normales.

Se obtienen los puntajes de las encuestas de autopercepción de la voz: VRQOL $=50, \mathrm{VHI}-10=40$ y autopercepción =pobre (escala de 5 categorías). El paciente completa 10 sesiones de terapia fonoaudiológica, tras lo cual mejoran sus puntajes de VRQOL $=34, V H I-10=21$, con autopercepción aún pobre. Al control el paciente persiste con disfonía, y a la nasofaringolaringoscopía se aprecia inmovilidad de la cuerda vocal izquierda en posición lateral e importante hiato fonatorio, por lo que se realiza en forma ambulatoria una laringoplastía de inyección con ácido hialurónico $\left(\right.$ Restylane $\left.^{\circledR}\right)$ tres semanas posterior al inicio del cuadro. Al igual que en el primer caso, el procedimiento se realizó bajo visión nasofibroscópica con paciente vigil y anestesia local, y se utilizó la técnica de inyección percutánea con abordaje transtirohioideo, logrando cierre completo del hiato fonatorio. Se realiza control 3 días posterior a la laringoplastía de inyección. La nasofaringolaringoscopía se informa con cuerda vocal izquierda inmóvil en posición paramediana, movilidad de cuerda vocal derecha conservada en aducción y abducción. Se observa mínimo hiato fonatorio posterior. Comisura posterior normal. Actualmente paciente se encuentra realizando terapia fonoaudiológica. Figura 2.

\section{DISCUSIÓN}

La disfonía es un síntoma que puede presentarse luego de una intubación endotraqueal, incluso en cirugías no relacionadas con patologías de cabeza y cuello ${ }^{1}$ como en los casos presentados. La incidencia reportada varía ampliamente, presentándose hasta en $71 \%$ de los pacientes sometidos a anestesia general ${ }^{6}$. Ésta puede deberse a distintas causas, como lesiones de la mucosa de la cuerda vocal, granulomas vocales o luxación de la articulación cricoaritenoidea. De manera muy infrecuente se han documentado casos en la literatura en los que la disfonía es causada por una parálisis cordal unilateral'1.

La parálisis cordal posintubación endotraqueal es una complicación poco frecuente ${ }^{1}$. Si bien exis- 
ten casos reportados de parálisis cordal bilateral posterior a anestesia general ${ }^{7,8}$, la gran mayoría de los pacientes presenta una parálisis unilateral, siendo comprometida la cuerda vocal izquierda en el $67 \%$ de los $\operatorname{casos}^{3,9}$.

Kikura y col. en una serie de 24 pacientes, reportó que la disfonía secundaria a una parálisis de cuerda vocal posoperatoria se presentaba dentro de las primeras 24 horas posteriores a la intubación, sin embargo, el diagnóstico de parálisis cordal se realizó en promedio a los 14 días, con un rango de 1 a 30 días $^{3}$.

En la literatura se han descrito diversos factores asociados a esta complicación. En cuanto a los factores asociados al paciente, se observa que la incidencia de este cuadro aumenta con la edad. En un análisis multivariado, se vio que el riesgo se incrementa 3 veces en aquellos mayores de 50 años ${ }^{3}$. Los antecedentes mórbidos de los pacientes también resultan relevantes. El riesgo se duplica en los pacientes que padecen de diabetes miellitus e hipertensión arterial ${ }^{3}$.

En cuanto a los factores asociados al procedimiento, se ha visto que la incidencia de esta complicación aumenta con la duración de la anestesia general, siendo particularmente mayor el riesgo, si se prolonga por más de 6 horas ${ }^{3}$. Sin embargo, se han presentado casos en los que la cirugía fue de corta duración, de hasta 150 minutos $^{2}$.

El tubo orotraqueal (TOT) debe quedar bien posicionado, y se debe evitar que se movilice durante la cirugía ${ }^{1,2}$. Kikura y col. no logró mostrar asociación entre el tamaño del TOT y el desarrollo de parálisis cordal ${ }^{3}$. Se debe tener en consideración que no en todos los pacientes existe el antecedente de intubación difícil ${ }^{1,9}$.

Además de la disfonía, la parálisis cordal puede asociarse a un trastorno de la deglución, constituyendo un factor de riesgo de aspiración, pudiendo ser una complicación severa ${ }^{10}$.

En cuanto a la patogenia de esta complicación, Ellis y Pallister realizaron disección en cadáveres, y describieron el trayecto del nervio laríngeo recurrente y sus dos ramas. La rama anterior discurre medial a la lámina del cartílago tiroideo, inervando los músculos cricoaritenoideo lateral y tiroaritenoideo, siendo susceptible de ser lesionada por compresión durante la intubación².
Por otro lado, la rama posterior inerva los músculos cricoaritenoideo posterior e interaritenoideo, presentando un trayecto con menor probabilidad de lesión.

El mecanismo más frecuente de daño sobre el nervio laríngeo recurrente durante la intubación endotraqueal es la neuropraxia. Se ha sugerido que la neuropraxia por presión sobre la rama anterior del nervio laríngeo recurrente se produce si el manguito es inflado a nivel o justo por debajo de las cuerdas vocales, así como si es insuficientemente desinflado previo a la extubación ${ }^{2}$. Se ha descrito como posible causa de parálisis de cuerda vocal la luxación traumática e inflamación cricoaritenoidea al momento de posicionar la cabeza del paciente previo a la intubación ${ }^{11}$. La cuerda vocal paralizada tiende a adoptar una posición lateral, lo cual se traduce en insuficiencia glótica ${ }^{12}$.

La dislocación aritenoidea es una causa posible de disfonía prolongada en el posoperatorio, secundaria a hipomovilidad de las cuerdas vocales. La dislocación se produce en intubaciones traumáticas. Resulta difícil diferenciar entre la dislocación aritenoidea y la parálisis cordal debido a su presentación similar. La ausencia del signo del "empujón" (jostle sign) asociado a signos de traumatismos de la zona es un indicador consistente de dislocación aritenoidea ${ }^{13}$.

En el seguimiento de 36 pacientes afectados por parálisis cordal posintubación, se evidenció que la movilidad cordal regresa a la normalidad en un plazo de hasta 1 año posterior a su inicio, en el $58 \%$ de los casos ${ }^{1}$.

Tradicionalmente, el manejo inicial de estos pacientes ha consistido en la observación en espera de la recuperación espontánea de la movilidad cordal o de la compensación, o la terapia fonoaudiológica para favorecer la recuperación de la función ${ }^{14}$.

Debido al escaso número de pacientes con parálisis cordal posintubación, no existen estudios que evalúen cuál es el tratamiento de elección en este subgrupo.

En los últimos años se ha introducido la laringoplastía de inyección temporal de la cuerda vocal como otra opción en el manejo inicial de los pacientes con parálisis unilateral de cuerda vocal, cuyo objetivo es disminuir temporalmente la insu- 
ficiencia glótica en espera de la recuperación de la movilidad o la compensación ${ }^{15}$.

La laringoplastía de inyección consiste en lograr, mediante la inyección de un agente reabsorbible, la medialización de la cuerda vocal inmóvil, dejándola en una posición más favorable para el cierre glótico ${ }^{16,17}$. Para esto se han descrito diferentes técnicas como laringoscopía directa y anestesia general 0 en paciente despierto la cual puede ser transoral o percutánea. La técnica que se realiza en nuestro centro es percutánea con paciente despierto. Esta técnica requiere de 2 personas. Se realiza con anestesia traqueal (lidocaína $2 \mathrm{cc}$ al $4 \%$ ) y con la tos del paciente se nebuliza la lidocaína actuando sobre cuerdas vocales. Luego por visión videonasofibroscópica y punción percutánea a nivel transtiroideo, transtirohioideo 0 transcricotiroideo, se inyecta material de relleno, en este caso ácido hialurónico, inmediatamente anterior a la apófisis vocal y lateral a la línea arcuata provocando un efecto en la voz en forma inmediata.

Actualmente existe una preferencia por los materiales de inyección temporales (que se reabsorben en un lapso de tiempo de entre 3 y 6 meses), ya que permiten la reversibilidad en caso de recuperación de la movilidad cordal, y la posibilidad de inyecciones sucesivas en el tiempo en caso contrario. Además, existe evidencia de que los pacientes con parálisis unilateral de cuerda vocal que son sometidos precozmente ( $<6$ meses) a una inyección de medialización con un material temporal tienen menor probabilidad de requerir un procedimiento definitivo que los pacientes que inicialmente fueron manejados con observación 0 terapia fonoaudiológica ${ }^{9,10,18}$.

Mor y col recientemente, definieron una serie de factores clínicos de mal pronóstico para pacientes con parálisis cordal unilateral, definido como necesidad de laringoplastía de inyección. Estos son: pacientes jóvenes, compromiso de cuerda vocal izquierda, etiología neoplásica 0 iatrogénica, 90 días o más desde el inicio del cuadro, y aspiración. Por otro lado, aquellos con parálisis cordal derecha, menos de 90 días de iniciado el cuadro, causa idiopática y uso de inyección de aumento, podría evitar la necesidad de laringoplastía de inyección ${ }^{19}$.
Tomando en consideración estos elementos, el clínico debe decidir de manera racional el momento oportuno y tipo de terapia.

Múltiples esfuerzos en investigación, se han realizado, para obtener materiales biocompatibles con características óptimas, para utilizar en cirugía de aumento en diferentes especialidades. La inyección de aumento con ácido hialurónico $\left(\right.$ Restylane $^{\circledR}$ ) ha mostrado ser una técnica efectiva en el tratamiento de pacientes con disfonía por parálisis cordal unilateral. Aquellos pacientes sometidos a esta modalidad terapéutica presentan mejoría en las escalas de evaluación subjetiva de la voz ${ }^{4,7,8,10}$, así como en el tiempo fonatorio ${ }^{8}$. Además, es posible objetivar mediante videoestrobolaringoscopía, el cierre del hiato fonatorio $0^{8,10}$. Si bien esta sustancia, se mantiene por un plazo máximo de 6 meses en las cuerdas vocales $^{19}$, los beneficios pueden verse hasta 12 meses luego del procedimiento ${ }^{8}$. Aquellos pacientes con insuficiencia glótica menor a $1 \mathrm{~mm}$ son buenos candidatos para este tratamiento. Además, el uso de Restylane ${ }^{\circledR}$ facilita la terapia fonoaudiológica8 . $^{2}$

El uso de Restylane ${ }^{\circledR}$, u otros materiales en base a ácido hialurónico, no alteran las propiedades viscoelásticas de las cuerdas vocales, ni su capacidad vibratoria ${ }^{20}$. Su aplicación en las cuerdas vocales de modelos animales, atrae fibroblastos, generando colágeno nuevo, lo cual resulta en un aumento del tejido blando de manera endógena. La generación de tejido nuevo por el propio hospedero, genera un efecto duradero, sin reacciones inflamatorias adversas ${ }^{20}$.

Los efectos adversos relacionados al uso de ácido hialurónico se explican por su alto contenido en proteínas animales, generando reacciones de hipersensibilidad. Sin embargo, se presentan en el $0,06 \%$ de los pacientes que reciben inyección de aumento con Restylane ${ }^{\circledR 21}$.

Se presentan dos casos de parálisis cordal unilateral izquierda posintubación endotraqueal, en relación a cirugías abdominales. Los pacientes fueron manejados con una laringoplastía de inyección con ácido hialurónico (Restylane ${ }^{\circledR}$ ), en conjunto con terapia fonoaudiológica, obteniendo buenos resultados vocales, con seguimiento de dos años para el primer caso. 


\section{REFERENCIAS}

1. Cavo JW JR. True vocal cord paralysis following intubation. Laryngoscope 1985; 95(11): 1352-9.

2. ElLIS PD, PalLISTER WK. Recurrent laryngeal nerve palsy and endotracheal intubation. $J$ Laryngol Otol 1975; 89(8): 823-6.

3. Kikura M, Suzuki K, Itagaki T, Takada T, Sato S. Age and comorbidity as risk factors for vocal cord paralysis associated with tracheal intubation. $\mathrm{Br}$ $J$ Anaesth 2007; 98(4): 524-30.

4. Young VN, Smith LJ, Rosen C. Voice outcome following acute unilateral vocal fold paralysis. Ann Otol Rhinol Laryngol 2013; 122(3): 197-204.

5. Vinson KN, Zraick RI, Ragland FJ. Injection versus medialization laryngoplasty for the treatment of unilateral vocal fold paralysis: follow-up at six months. Laryngoscope 2010; 120(9): 1802-7.

6. Jones MW, Catling S, Evans E, Green DH, Green JR. Hoarseness after tracheal intubation. Anaesthesia 1992; 47(3): 213-6.

7. Nama RK, Bhosale GP, Butala BP, Sharma AR. Bilateral adductor vocal cord palsy: complication of prolonged intraoperative hypotension after endotraqueal intubation. Middle East $J$ Anaesthesiol 2015; 23(3): 339-42.

8. WASON R, GuPTA P, Gogia AR. Bilateral adductor vocal cord paresis following endotracheal intubation for general anaesthesia. Anaesth Intensive Care 2004; 32(3): 417-8.

9. Lu YH, Hsien MW, Tong YH. Unilateral vocal cord paralysis following endotracheal intubation--a case report. Acta Anaesthesiol Sin 1999; 37(4): 221-4.

10. Hamdan AL, Moukarbel RV, Farhat F, Obeid M. Vocal cord paralysis after open-heart surgery. Eur $J$ Cardiothorac Surg 2002; 21(4): 671-4.

11. Colton House J, NoordziJ JP, Murgia B, Langmore $S$. Laryngeal injury from prolonged intubation: a prospective analysis of contributing factors. Laryngoscope 2011; 121(3): 596-600.
12. Bielamowicz S, Stager SV. Diagnosis of unilateral recurrent laryngeal nerve paralysis: laryngeal electromyography, subjective ratingscales, acoustic and aerodynamic measures. Laryngoscope 2006; 116(3): 359-64.

13. Goz V, QuRESHI S, HeCHT AC. Arytenoid dislocation as a cause of prolonged hoarseness after cervical discectomy and fusion. Global Spine $\mathrm{J}$ 2013; $3(1): 47-50$.

14. TUCKER HM. Vocal cord paralysis-etiology and management. Laryngoscope 1980; 90: 58590.

15. Sulica L, Rosen CA, Postma GN, et al. Current practice in injection augmentation of the vocal folds: indications, treatment principles, techniques and complications. Laryngoscope 2010; 120: 319-25.

16. Clary MS, Milam BM, Courey M. Office based vocal fold injection with the laryngeal introducer technique. Laryngoscope 2014; 124 (9): 2114-7.

17. MODI, VIKASH K. Vocal fold injection medialization laryngoplasty. Advances in Oto Rhino Laryngology 2012; 73: 9.

18. Yung KC, Likhterov I, Courey MS. Effect of temporary vocal fold inyection medialization on the rate of permanent medialization laryngoplasty in unilateral vocal fold paralysis patients. Laryngoscope 2011; 121: 2191-4.

19. Mor N, Wu G, Aylward A, Christos PJ, Sulica L. Predictors for Permanent Medialization Laryngoplasty in Unilateral Vocal Fold Paralysis. Otolaryngol Head Neck Surg. 2016 May 3.

20. Borzacchiello A, Mayol L, Gärskog O, Dahlovist A, AmBrosio $\mathrm{L}$. Evaluation of injection augmentation treatment of hyaluronic acid based materials on rabbit vocal folds viscoelasticity. J Mater Sci Mater Med 2005; 16(6): 553-7.

21. Friedman PM, Mafong EA, Kauvar an, Geronemus RG. Safety data of injectable nonanimal stabilized hyaluronic acid gel for soft tissue augmentation. Dermatol Surg 2002; 28(6): 491-4.

\footnotetext{
Dirección: Carla Napolitano V.

Departamento de Otorrinolaringología, Pontificia Universidad Católica de Chile

E mail: cnapolitanov@gmail.com
} 